\title{
Type II half logistic Ibrahim distribution with applications
}

\author{
I. B. Abdul-Moniem ${ }^{1 *}$, M. Seham² \\ ${ }^{I}$ Department of Statistics, Higher Institute of Management in Sohag, Sohag, Egypt \\ ${ }^{2}$ Department of Mathematics, Faculty of science, Aswan University, Aswan, Egypt \\ *Corresponding author E-mail:ibtaib@hotmail.com
}

\begin{abstract}
In this paper, we introduce a new distribution called type II half logistic Ibrahim (TIIHLI) distribution. Some properties of this distribu-
\end{abstract} tion will be discussed. The estimation of unknown parameters for TIIHLI distribution will be handled using real data.

Keywords: Ibrahim Distribution; Type II Half Logistic Distributions; Marshall-Olkin Distribution; Moments, Parameter Estimation.

\section{Introduction}

A new family of distributions called the type II half logistic (TIIHL) distributions is introduced and studied by Hassan et al. [3]. The cumulative distributions function (cdf) of TIIHL distributions is

$$
F(x)=\frac{2[G(x ; \xi)]^{\lambda}}{1+[G(x ; \xi)]^{2}} ; \quad x>0 \text { and } \lambda>0,
$$

Where, $\lambda$ is a scale parameter and $G(x ; \xi)$ is a baseline cdf, which depends on a parameter vector $\zeta$.

The probability density function (pdf) is given by

$$
f(x)=\frac{2 \lambda g(x ; \xi)[G(x ; \xi)]^{\lambda-1}}{\left\{1+[G(x ; \xi)]^{\lambda}\right\}^{2}} ; \quad x>0 \text { and } \lambda>0,
$$

Where $g(x ; \xi)$ is the pdf of base distribution.

The survival function $\bar{F}(x)$, hazard rate function $h(x)$ and reverse hazard rate function $h^{*}(x)$ are, respectively, given by

$$
\begin{aligned}
& \bar{F}(x)=\frac{1-[G(x ; \xi)]^{\lambda}}{1+[G(x ; \xi)]^{\lambda}} ; \quad x>0 \text { and } \lambda>0, \\
& h(x)=\frac{2 \lambda g(x ; \xi)[G(x ; \xi)]^{\lambda-1}}{1-[G(x ; \xi)]^{2 \lambda}} ; \quad x>0 \text { and } \lambda>0,
\end{aligned}
$$

and

$$
h^{*}(x)=\frac{\lambda g(x ; \xi)}{G(x ; \xi)\left\{1+[G(x ; \xi)]^{\lambda}\right\}}=\frac{\lambda h_{1}^{*}(x)}{1+[G(x ; \xi)]^{\lambda}} ; \quad x>0 \text { and } \lambda>0 \text {, }
$$

Where $h_{1}^{*}(x)$ is the reverse hazard rate function of base distribution. More details on the family of TIIHL distributions see Hassan et al. [3]. 
Lemma: The TIIHL distribution at $\lambda=1$ tends to Marshall-Olkin (M-O) distribution with $\alpha=0.5$

Proof: Put $\lambda=1$ in (1), we get

$$
F(x)=\frac{2 G(x ; \xi)}{1+G(x ; \xi)}
$$

The cdf of M-O distribution (Marshall and Olkin [4]) is

$$
F(x)=\frac{G(x ; \xi)}{1-\bar{\alpha} \bar{G}(x ; \xi)} ; \quad \bar{\alpha}=1-\alpha .
$$

Set $\alpha=0.5$ in (7) and after simplified, we get (6). $\square$

A random variable $X$ has Ibrahim distribution with two parameters $\beta$ and $\theta$ if its probability density function (pdf) is (Abdul-Moniem [1])

$$
g(x)=\frac{\beta}{\theta+1}\left[e^{-\beta x}+2 \theta \beta x e^{-(\beta x)^{2}}\right] ; \quad x \geq 0,(\beta \text { and } \theta>0) .
$$

The cdf corresponding (8) is

$$
G(x)=1-\frac{1}{\theta+1}\left(e^{-\beta x}+\theta e^{-(\beta x)^{2}}\right) ; \quad x \geq 0,(\beta \text { and } \theta>0) .
$$

For properties of this distribution see Abdul-Moniem [1].

The rest of the paper is organized as follows. In Section 2 we introduce TIIHL distribution. The traditional moments for TIIHL distribution are given in Section 3. In Section 4 we discussed the parameters estimators using maximum likelihood method. We use a real data set to show that the TIIHL distribution can be a better model than one based on the Ibrahim distribution in Section 5.

\section{Type II half logistic Ibrahim distribution}

Using (1) and (9), we can define the cdf of type II half logistic Ibrahim (TIIHLI) distribution as follows

$$
F(x)=\frac{2[G(x)]^{\lambda}}{1+[G(x)]^{\lambda}} ; \quad x \geq 0,(\theta, \beta \text { and } \lambda>0) .
$$

The pdf of TIIHLI distribution is

$$
f(x)=\frac{2 \lambda \beta\left[e^{-\beta x}+2 \theta \beta x e^{-(\beta x)^{2}}\right][G(x)]^{\lambda-1}}{(\theta+1)\left\{1+[G(x)]^{\lambda}\right\}^{2}} ; \quad x \geq 0,(\theta, \beta \text { and } \lambda>0) \text {, }
$$

The survival function $\bar{F}(x)$, hazard rate function $h(x)$ and reverse hazard rate function $h^{*}(x)$ corresponding (11) are, respectively, given by

$$
\begin{aligned}
& \bar{F}(x)=\frac{1-[G(x)]^{\lambda}}{1+[G(x)]^{\lambda}} ; \quad x \geq 0,(\theta, \beta \text { and } \lambda>0) . \\
& h(x)=\frac{2 \lambda \frac{\beta}{\theta+1}\left[e^{-\beta x}+2 \theta \beta x e^{-(\beta x)^{2}}\right][G(x)]^{\lambda-1}}{1-[G(x)]^{2 \lambda}} ; \quad x \geq 0,(\theta, \beta \text { and } \lambda>0) .
\end{aligned}
$$

and

$$
h^{*}(x)=\frac{\lambda \beta\left[e^{-\beta x}+2 \theta \beta x e^{-(\beta x)^{2}}\right][G(x)]^{-1}}{(\theta+1)\left\{1+[G(x)]^{2}\right\}} ; \quad x \geq 0,(\theta, \beta \text { and } \lambda>0) \text {. }
$$

Where $G(x)$ is given by (9).

Figures 1, 2 and 3 depict the behavior of the distribution for some parameter values. 


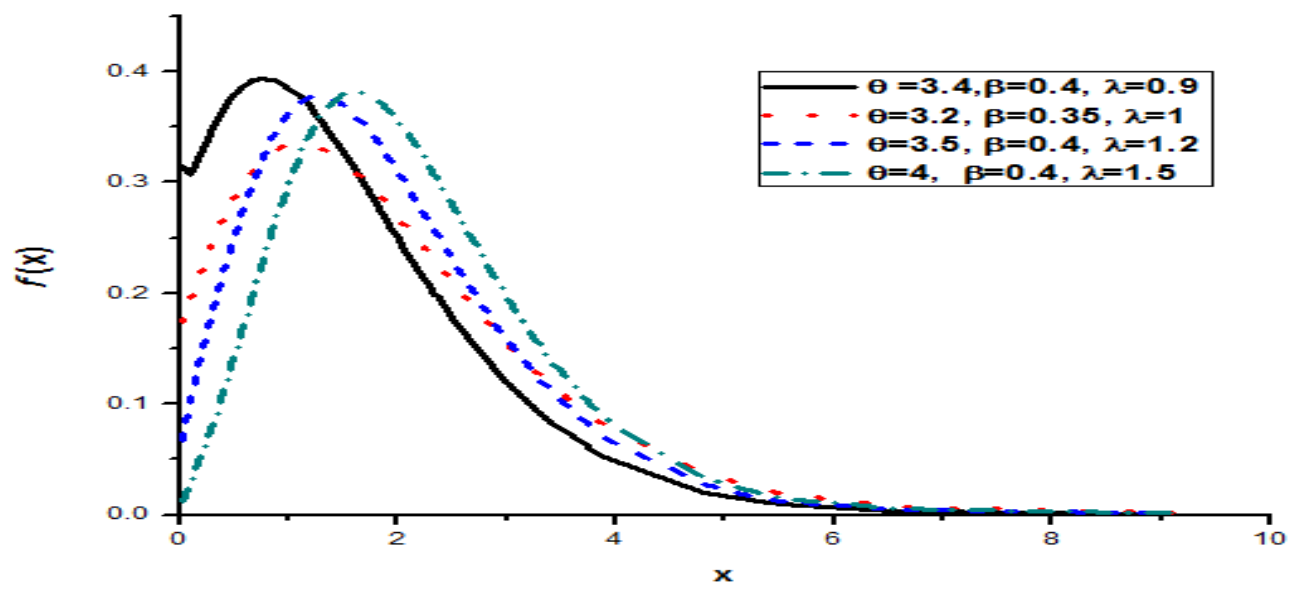

Fig. 1: Plots of PDF

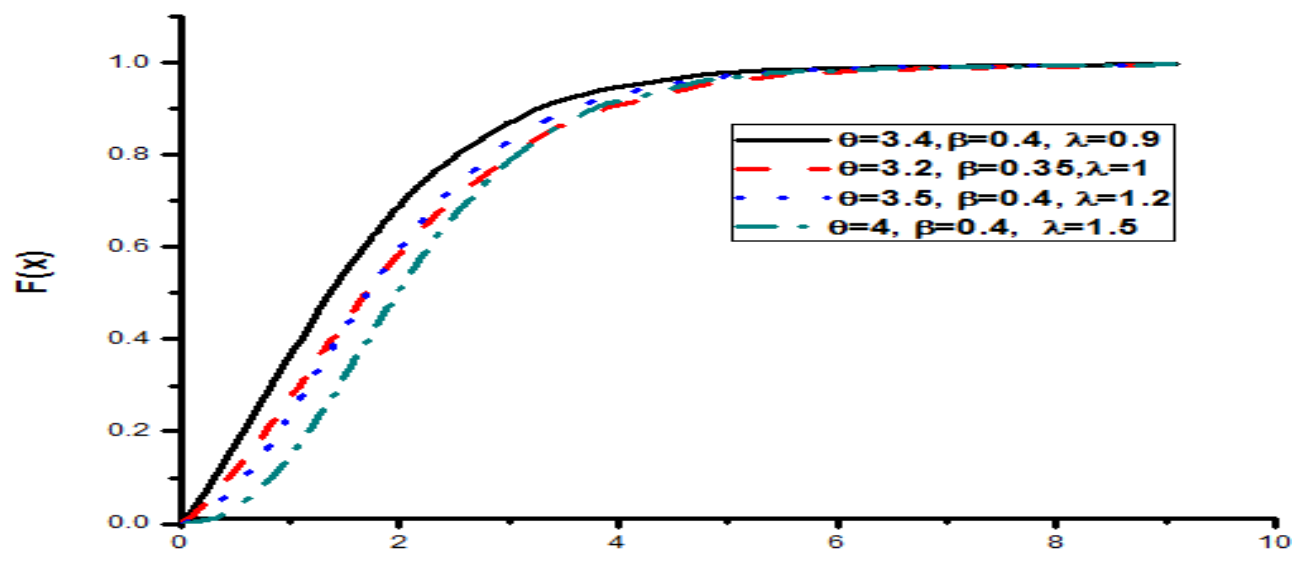

Fig. 2: Plots of $\stackrel{\mathbf{x}}{\mathrm{CDF}}$

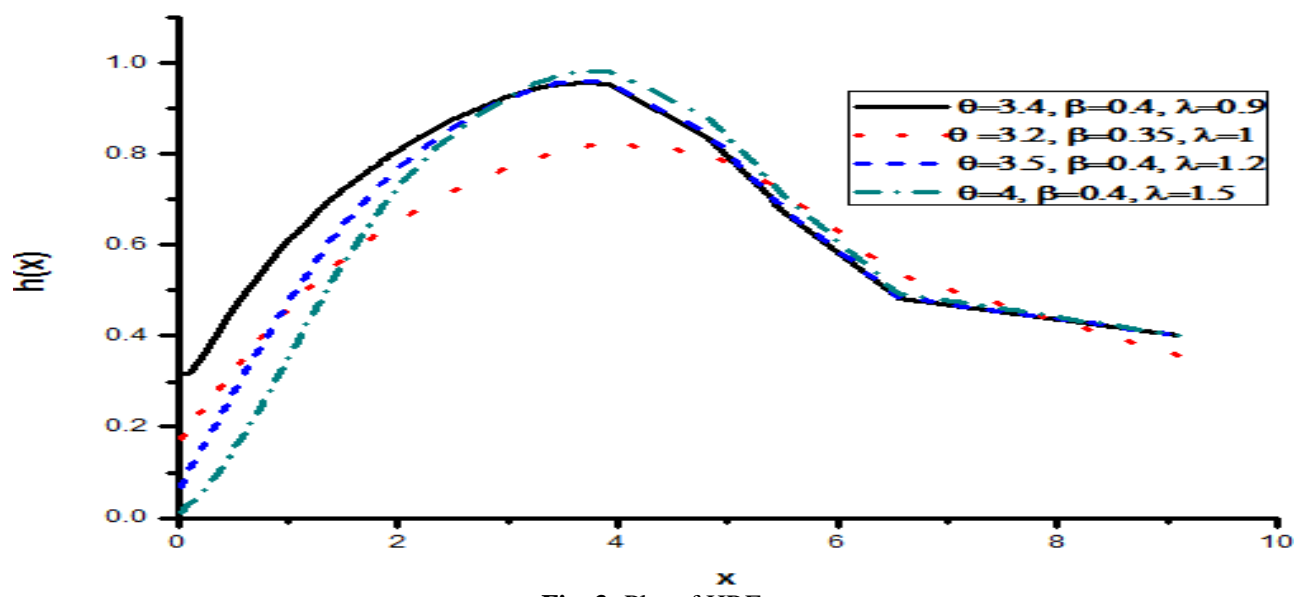

Fig. 3: Plot of $\mathrm{x}$

\section{Statistical properties}

In this section some statistical properties of TIIHLI distribution are discussed.

\subsection{Moments}

The $\mathrm{r}^{\text {th }}$ traditional moments for TIIHLI distribution is 


$$
\begin{aligned}
& \mu_{r}^{\prime}=\frac{2 \lambda \beta}{(\theta+1)} \int_{0}^{\infty} \frac{x^{r}\left[e^{-\beta x}+2 \theta \beta x e^{-(\beta x)^{2}}\right]\left[1-\frac{1}{\theta+1}\left(e^{-\beta x}+\theta e^{-(\beta x)^{2}}\right)\right]^{\lambda-1}}{\left\{1+\left[1-\frac{1}{\theta+1}\left(e^{-\beta x}+\theta e^{-(\beta x)^{2}}\right)\right]^{2}\right\}^{2}} d x \\
& =\frac{2 \lambda \beta}{(\theta+1)} \sum_{i=0}^{\infty}(-1)^{i}(i+1) \int_{0}^{\infty} x^{r}\left[e^{-\beta x}+2 \theta \beta x e^{-(\beta x)^{2}}\right]\left[1-\frac{1}{\theta+1}\left(e^{-\beta x}+\theta e^{-(\beta x)^{2}}\right)\right]^{\lambda(i+1)-1} d x \\
& =2 \lambda \beta \sum_{i=0}^{\infty} \sum_{j=0}^{\infty} \frac{(-1)^{i+j}(i+1)}{(\theta+1)^{j+1}}\left(\begin{array}{c}
\lambda(i+1)-1 \\
j
\end{array}\right) \int_{0}^{\infty} x^{r}\left[e^{-\beta x}+2 \theta \beta x e^{-(\beta x)^{2}}\right]\left(e^{-\beta x}+\theta e^{-(\beta x)^{2}}\right)^{j} d x \\
& =2 \lambda \beta \sum_{i=0}^{\infty} \sum_{j=0}^{\infty} \sum_{k=0}^{j} \frac{(-1)^{i+j}(i+1) \theta^{j}}{(\theta+1)^{k+1}}\left(\begin{array}{l}
j \\
k
\end{array}\right)\left(\begin{array}{l}
\lambda(i+1)-1 \\
j
\end{array}\right) \\
& \int_{0}^{\infty} x^{r}\left[e^{-\beta x}+2 \theta \beta x e^{-(\beta x)^{2}}\right] e^{-\beta(j-k) x-k(\beta x)^{2}} d x \\
& =2 \lambda \beta \sum_{i=0}^{\infty} \sum_{j=0}^{\infty} \sum_{k=0}^{j} \frac{(-1)^{i+j}(i+1) \theta^{j}}{(\theta+1)^{j+1}}\left(\begin{array}{l}
j \\
k
\end{array}\right)\left(\begin{array}{l}
\lambda(i+1)-1 \\
j
\end{array}\right)\left\{I_{1}+2 \theta \beta I_{2}\right\}
\end{aligned}
$$

\section{Where}

$I_{1}=\int_{0}^{\infty} x^{r} e^{-\beta(j+1-k) x-k(\beta x)^{2}} d x$

and

$I_{2}=\int_{0}^{\infty} x^{r+1} e^{-\beta(j-k) x-(k+1)(\beta x)^{2}} d x$

$I_{1}=\int_{0}^{\infty} x^{r} e^{-\beta(j+1-k) x-k(\beta x)^{2}} d x$

Let $y=\beta(j+1-k) x+k(\beta x)^{2} \Rightarrow x=\frac{\sqrt{4 k y+(j+1-k)^{2}}-(j+1-k)}{2 \beta k}$

$$
\begin{aligned}
& \text { and } d x=\frac{d y}{\beta \sqrt{4 k y+(j+1-k)^{2}}} \\
& \begin{aligned}
I_{1} & =\frac{1}{(2 \beta k)^{r}} \int_{0}^{\infty}\left[\sqrt{4 k y+(j+1-k)^{2}}-(j+1-k)\right]^{r} e^{-y} \frac{d y}{\beta \sqrt{4 k y+(j+1-k)^{2}}} \\
& =\frac{2 k}{(2 \beta k)^{r+1}} \sum_{s=0}^{r}(-1)^{s}\left(\begin{array}{l}
r \\
s
\end{array}\right)(j+1-k)^{s} \int_{0}^{\infty}\left[\sqrt{4 k y+(j+1-k)^{2}}\right]^{r-s-1} e^{-y} d y \\
& =\frac{2 k}{(2 \beta k)^{r+1}} \sum_{s=0}^{r} \sum_{m=0}^{\infty}(-1)^{s}\left(\begin{array}{l}
r \\
s
\end{array}\right)\left(\begin{array}{c}
\frac{r-s-1}{2} \\
m
\end{array}\right)(j+1-k)^{s+m}(4 k)^{\frac{r-s-1}{2}-m} \int_{0}^{\infty} y y^{\frac{r-s-1}{2}-m} e^{-y} d y \\
& =\frac{2 k}{(2 \beta k)^{r+1}} \sum_{s=0}^{r} \sum_{m=0}^{\infty}(-1)^{s}\left(\begin{array}{l}
r \\
s
\end{array}\right)\left(\begin{array}{c}
\frac{r-s-1}{2} \\
m
\end{array}\right)(j+1-k)^{s+m}(4 k)^{\frac{r-s-1}{2}-m} \Gamma\left(\frac{r-s+1}{2}-m\right)
\end{aligned}
\end{aligned}
$$

$I_{2}=\int_{0}^{\infty} x^{r+1} e^{-\beta(j-k) x-(k+1)(\beta x)^{2}} d x$

$$
\begin{aligned}
& \qquad y=\beta(j-k) x+(k+1)(\beta x)^{2} \Rightarrow x=\frac{\sqrt{4(k+1) y+(j-k)^{2}}-(j-k)}{2 \beta(k+1)} \\
& \text { Let } \\
& \text { and } d x=\frac{d y}{\beta \sqrt{4(k+1) y+(j-k)^{2}}}
\end{aligned}
$$




$$
\begin{aligned}
I_{2} & =\frac{1}{[2 \beta(k+1)]^{r+1}} \int_{0}^{\infty}\left[\sqrt{4(k+1) y+(j-k)^{2}}-(j-k)\right]^{r+1} e^{-y} \frac{d y}{\beta \sqrt{4(k+1) y+(j-k)^{2}}} \\
& =\frac{1}{\beta[2 \beta(k+1)]^{r+1}} \sum_{l=0}^{r+1}(-1)^{l}\left(\begin{array}{c}
r+1 \\
l
\end{array}\right)(j-k)^{l} \int_{0}^{\infty}\left[\sqrt{4(k+1) y+(j-k)^{2}}\right]^{r-l} e^{-y} d y \\
& =\frac{1}{\beta[2 \beta(k+1)]^{r+1}} \sum_{l=0}^{r+1} \sum_{n=0}^{\infty}(-1)^{l}\left(\begin{array}{c}
r+1 \\
l
\end{array}\right)\left(\begin{array}{c}
r-l \\
2 \\
n
\end{array}\right)(j-k)^{l+2 n}[4(k+1)]^{\frac{r-l}{2}-n} \int_{0}^{\infty} y^{\frac{r-l}{2}-n} e^{-y} d y \\
I_{2} & =\frac{1}{\beta[2 \beta(k+1)]^{r+1}} \sum_{l=0}^{r+1} \sum_{n=0}^{\infty}(-1)^{l}\left(\begin{array}{c}
r+1 \\
l
\end{array}\right)\left(\begin{array}{c}
r-l \\
2 \\
n
\end{array}\right)(j-k)^{l+2 n}[4(k+1)]^{\frac{r-l}{2}-n} \Gamma\left(\frac{r-l}{2}-n+1\right)
\end{aligned}
$$

Substituting from (16) and (17) in (15), we get

$$
\begin{gathered}
\mu_{r}^{\prime}=2 \lambda \beta \sum_{i=0}^{\infty} \sum_{j=0}^{\infty} \sum_{k=0}^{j} \frac{(-1)^{i+j}(i+1) \theta^{j}}{(\theta+1)^{j+1}}\left(\begin{array}{c}
j \\
k
\end{array}\right)\left(\begin{array}{c}
\lambda(i+1)-1 \\
j
\end{array}\right)\left\{\frac{1}{\beta^{r+1}} \sum_{s=0}^{r} \sum_{m=0}^{\infty}(-1)^{s}\left(\begin{array}{c}
r \\
s
\end{array}\right)\left(\begin{array}{c}
\frac{r-s-1}{2} \\
m
\end{array}\right) \frac{(j+1-k)^{s+m}}{2^{s+1} k^{\frac{r+s+1}{2}+m}} \Gamma\left(\frac{r-s+1}{2}-m\right)\right. \\
\left.+\frac{\theta}{\beta^{r+1}} \sum_{l=0}^{r+1} \sum_{n=0}^{\infty}(-1)^{\prime}\left(\begin{array}{c}
r+1 \\
l
\end{array}\right)\left(\begin{array}{c}
\frac{r-l}{2} \\
n
\end{array}\right) \frac{(j-k)^{l+2 n}}{2^{2 n+l+1}(k+1)^{\frac{r+2+l}{2}+n}} \Gamma\left(\frac{r-l}{2}-n+1\right)\right\}
\end{gathered}
$$

\subsection{The mode}

We can get the mode for TIIHLI distribution by solving the following equation

$$
\begin{aligned}
& \left\{(\theta+1)^{\lambda}+\left[\theta+1-\left(e^{-\beta x}+\theta e^{-(\beta x)^{2}}\right)\right]^{\lambda}\right\}\left\{(\theta+1)\left(-\beta e^{-\beta x}+2 \theta\left[\beta-2(\beta x)^{2}\right] e^{-(\beta x)^{2}}\right)\right\}+(\theta+1)^{\lambda} \\
& \left\{\beta \lambda e^{-2 \beta x}+\theta \beta\left[4 \beta x^{2}+(2 \beta \lambda+\lambda-2 \beta-1) x-1\right] e^{-\beta x-(\beta x)^{2}}+2 \theta^{2}\left[(\lambda+1)(\beta x)^{2}-\beta\right] e^{-2(\beta x)^{2}}\right\} \\
& \left.+(\beta \lambda-2 \lambda) e^{-2 \beta x}+2 \theta^{2}\left[(\lambda+1)(\beta x)^{2}-2 \lambda \beta(x)^{2}-\beta\right] e^{-2(\beta x)^{2}}\right\}+\left[\theta+1-\left(e^{-\beta x}+\theta e^{-(\beta x)^{2}}\right)\right]^{\lambda} \\
& \left\{\left[4 \theta(\beta x)^{2}+\theta \beta(2 \beta \lambda-2 \beta-1) x-\lambda \theta x(3 \beta+2)-\theta \beta\right] e^{-\beta x-(\beta x)^{2}}=0\right.
\end{aligned}
$$

\subsection{The median}

The median of TIIHLI distribution is the solving of the following equation

$$
e^{-\beta x_{0.5}}+\theta e^{-\left(\beta x_{0.5}\right)^{2}}=(\theta+1)\left[1-\left(\frac{1}{3}\right)^{\frac{1}{\lambda}}\right]
$$

\section{Parameters estimators}

In this section, we consider maximum likelihood estimators (MLE) of TIIHLI. Let ${ }^{x_{1}, x_{2}, \ldots, x_{n}}$ be a random sample of size $\mathrm{n}$ from TIIHLI, then the log-likelihood function $L L$ can be written as

$$
\begin{aligned}
& L L \propto n[\ln (\lambda)+\ln (\beta)-\ln (\alpha+1)]+\sum_{i=1}^{n} \ln \left(e^{-\beta x_{i}}+2 \alpha \beta x_{i} e^{-\left(\beta x_{i}\right)^{2}}\right) \\
& +(\lambda-1) \sum_{i=1}^{n} \ln \left[1-\frac{1}{\alpha+1}\left(e^{-\beta x_{i}}+\alpha e^{-\left(\beta x_{i}\right)^{2}}\right)\right]-2 \sum_{i=1}^{n} \ln \left\{1+\left[1-\frac{1}{\alpha+1}\left(e^{-\beta x_{i}}+\alpha e^{-\left(\beta x_{i}\right)^{2}}\right)\right]\right\}
\end{aligned}
$$

The normal equations become 


$$
\begin{aligned}
& \frac{\partial L L}{\partial \alpha}=\frac{-n}{\alpha+1}+\sum_{i=1}^{n} \frac{2 \beta x_{i} e^{-\left(\beta x_{i}\right)^{2}}}{e^{-\beta x_{i}}+2 \alpha \beta x_{i} e^{-\left(\beta x_{i}\right)^{2}}}+(\lambda-1) \sum_{i=1}^{n} \frac{e^{-\beta x_{i}}-e^{-\left(\beta x_{i}\right)^{2}}}{(\alpha+1)^{2}-(\alpha+1)\left(e^{-\beta x_{i}}+\alpha e^{-\left(\beta x_{i}\right)^{2}}\right)}-2 \sum_{i=1}^{n} \frac{\lambda\left(e^{-\beta x_{i}}-e^{-\left(\beta x_{i}\right)^{2}}\right)\left[1-\frac{1}{\alpha+1}\left(e^{-\beta x_{i}}+\alpha e^{-\left(\beta x_{i}\right)^{2}}\right)\right]^{\lambda-1}}{(\alpha+1)^{2}+(\alpha+1)^{2}\left[1-\frac{1}{\alpha+1}\left(e^{-\beta x_{i}}+\alpha e^{-\left(\beta x_{i}\right)^{2}}\right)\right]^{2}} \\
& \frac{\partial L L}{\partial \beta}=\frac{n}{\beta}-\sum_{i=1}^{n} \frac{x_{i} e^{-\beta x_{i}}-2 \alpha x_{i}\left[1-2\left(\beta x_{i}\right)^{2}\right] e^{-\left(\beta x_{i}\right)^{2}}}{e^{-\beta x_{i}}+2 \alpha \beta x_{i} e^{-\left(\beta x_{i}\right)^{2}}}+(\lambda-1) \sum_{i=1}^{n} \frac{\left(x_{i} e^{-\beta x_{i}}+2 \alpha \beta\left(x_{i}\right)^{2} e^{-\left(\beta x_{i}\right)^{2}}\right)}{\alpha+1-\left(e^{-\beta x_{i}}+\alpha e^{-\left(\beta x_{i}\right)^{2}}\right)} \\
& -2 \sum_{i=1}^{n} \frac{\lambda\left[1-\frac{1}{\alpha+1}\left(e^{-\beta x_{i}}+\alpha e^{-\left(\beta x_{i}\right)^{2}}\right)\right]^{\lambda-1}\left(x_{i} e^{-\beta x_{i}}+2 \alpha \beta\left(x_{i}\right)^{2} e^{-\left(\beta x_{i}\right)^{2}}\right)}{\alpha+1+(\alpha+1)\left[1-\frac{1}{\alpha+1}\left(e^{-\beta x_{i}}+\alpha e^{-\left(\beta x_{i}\right)^{2}}\right)\right]^{\lambda}}, \\
& \frac{\partial L L}{\partial \lambda}=\frac{n}{\lambda}+\sum_{i=1}^{n} \ln \left[1-\frac{1}{\alpha+1}\left(e^{-\beta x_{i}}+\alpha e^{-\left(\beta x_{i}\right)^{2}}\right)\right]-2 \sum_{i=1}^{n} \frac{\left[1-\frac{1}{\alpha+1}\left(e^{-\beta x_{i}}+\alpha e^{-\left(\beta x_{i}\right)^{2}}\right)\right]^{\lambda} \ln \left[1-\frac{1}{\alpha+1}\left(e^{-\beta x_{i}}+\alpha e^{-\left(\beta x_{i}\right)^{2}}\right)\right]}{1+\left[1-\frac{1}{\alpha+1}\left(e^{-\beta x_{i}}+\alpha e^{-\left(\beta x_{i}\right)^{2}}\right)\right]^{\lambda}} .
\end{aligned}
$$

The MLE of $\alpha, \theta$ and $\lambda$ can be obtain by solving the equations (21), (22), and (23) using $\frac{\partial \ln L}{\partial \alpha}=0, \frac{\partial \ln L}{\partial \theta}=0$ and $\frac{\partial \ln L}{\partial \lambda}=0$.

\section{Application of TIIHLI distribution}

In this section, we fit TIIHLI to real data set and compare the fitness with the Marshall-Olkin and Ibrahim distributions. The set of data represents the survival times (in days) of 72 guinea pigs infected with virulent tubercle bacilli, observed and reported by Bjerkedal [2]. In order to compare distributions, we consider the K-S (Kolmogorov-Smirnov) statistic, - 2logL, AIC (Akaike Information Criterion), AICC (Akaike Information Criterion Corrected), BIC (Bayesian Information Criterion). The best distribution corresponds to lower K-S,

\begin{tabular}{|c|c|c|c|c|c|c|c|c|}
\hline \multirow{2}{*}{ Model } & \multicolumn{3}{|l|}{ MLEs } & \multicolumn{5}{|c|}{ Measures } \\
\hline & $\hat{\theta}$ & $\hat{\boldsymbol{\beta}}$ & $\hat{\lambda}$ & $\mathrm{K}-\mathrm{S}$ & $-2 \mathrm{LL}$ & AIC & AICC & BIC \\
\hline TIIHLID & 0.203 & 0.046 & 0.809 & 0.1 & 503.397 & 509.397 & 509.75 & 516.227 \\
\hline M-OID & 0.399 & 0.054 & - & 0.141 & 505.64 & 509.64 & 509.814 & 514.193 \\
\hline ID & 0.19 & 0.078 & - & 0.161 & 508.509 & 512.509 & 512.683 & 517.063 \\
\hline
\end{tabular}
$-2 \log \mathrm{L}, \mathrm{AIC}, \mathrm{BIC}, \mathrm{AICC}$ statistics value.

Table 1:Maximum-Likelihood Estimates, K-S Statistics and -2LL, AIC, AICC and BIC Values for the A Random Sample of 72 Guinea Pigs Infected with Virulent Tubercle Bacilli

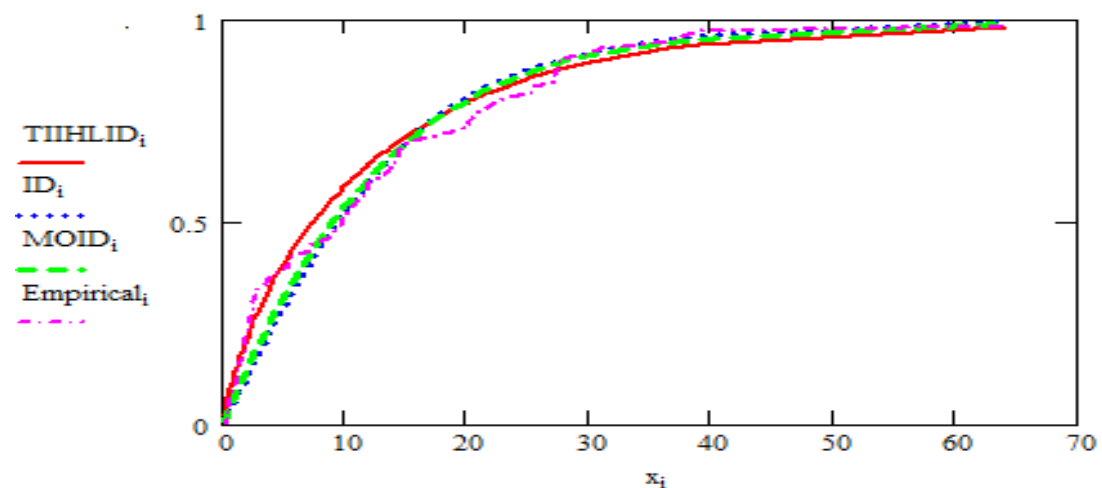

Fig. 4:Empirical, Fitted TIIHLID, ID and M-OID CDF of the Above Data.

\section{References}

[1] Abdul-Moniem, I. B., 2016. New Distribution. JP J. Fund. Appl. Stat., 10 (1 \& 2), 1-9.

[2] Bjerkedal, T., 1960. Acquisition of Resistance in Guinea Pies infected with Different Doses of Virulent Tubercle Bacilli. Amer. J. Hygiene, 72, 130-48.https://doi.org/10.1093/oxfordjournals.aje.a120129.

[3] Hassan, A. S., Elgarhy, M. and Shakil, M., 2017. Type II Half Logistic Family of Distributions with Applications. Pak. J. Stat. O. R., XIII (2), 245264.https://doi.org/10.18187/pjsor.v13i2.1560.

[4] Marshall,A. N, Olkin, I., 1997. A new method for adding a parameter to a family of distributions with applications to the exponential and Weibull families, Biometrica 84, 641-652.https://doi.org/10.1093/biomet/84.3.641. 